\title{
Alterações biopsicossociais decorrentes do isolamento social provocado pela pandemia da COVID-19 em alunos de Odontologia da Universidade de Pernambuco do campus Camaragibe: um estudo piloto
}

\author{
Biopsychosocial changes resulting from social isolation caused by the COVID-19 pandemic in \\ Dentistry students at the University of Pernambuco on the Camaragibe campus: a pilot study \\ Cambios biopsicosociales resultantes del aislamiento social causado por la pandemia COVID-19 en \\ estudiantes de Odontología de la Universidad de Pernambuco en el campus de Camaragibe: un \\ estudio piloto
}

Maria Eduarda Lemos Avelino ORCID: https://orcid.org/0000-0003-0153-5261 Universidade de Pernambuco, Brasil E-mail: mariaeduarda.avelino@upe.br Maria do Socorro Orestes Cardoso ORCID: https://orcid.org/0000-0001-9866-0899 Universidade de Pernambuco, Brasil E-mail: socorroorestes@yahoo.com.br José Afonso Milhomens Filho ORCID: https://orcid.org/0000-0002-7113-356X Universidade de Pernambuco, Brasil E-mail: afonso.milhomens@upe.br

Rosana Maria Coelho Travassos ORCID: https://orcid.org/0000-0003-4148-1288

Universidade de Pernambuco, Brasil E-mail: Rosana.travassos@upe.br

Maria Raquel Melo de Miranda Avelino ORCID: https://orcid.org/0000-0003-1075-9777 Universidade de Lisboa, Portugal E-mail: Raquelmavelino@gmail.com

Nathalia Melo de Medeiros ORCID: https://orcid.org/0000-0001-6487-1698 Universidade de Pernambuco, Brasil E-mail: nathaliammelo@hotmail.com Laura do Nascimento Arruda ORCID: https://orcid.org/0000-0002-9087-2157 Universidade de Pernambuco, Brasil E-mail: lauranarruda@gmail.com

Natália Gusmão Lobo

ORCID: https://orcid.org/0000-0003-3608-3242 Universidade de Pernambuco, Brasil

E-mail: nataliaagusmaolobo@gmail.com

\begin{abstract}
Resumo
Considerando-se a situação atual mundial, marcada por importantes crises na saúde pública e, mais recentemente, a pandemia causada pela COVID-19, o presente estudo teve como objetivo analisar as alterações biopsicossociais decorrentes do isolamento social provocado pela Pandemia em estudantes de Odontologia da Universidade de Pernambuco. No que se refere a metodologia tratou-se de um levantamento de campo (survey) de análise quantitativa, de corte transversal e descritivo. A amostra foi constituída de um universo de 379 alunos dos quais 106 participaram do estudo. A coleta foi realizada em um período de 72 horas, em junho de 2021, onde os alunos responderam um questionário on-line desenvolvido por meio da plataforma Google Forms contendo perguntas fechadas e abertas sobre dados sociodemográficos, bem como aspectos relacionados a alterações físicas e psicossociais. Os resultados sugerem que na amostra pesquisada o isolamento social teve repercussões na rotina diária, muitos relataram problemas de concentração, alterações de sono, sentimento de medo, preocupação e tristeza. Concluiu-se que a maioria dos pesquisados foi do gênero feminino, a faixa etária prevalente foi de 18 a 24 anos, moram com mais 2 ou 4 pessoas em
\end{abstract}


seus domicílios, são solteiros e relataram terem tido perdas financeiras com a pandemia. Entre as principais questões psicossociais destacaram-se: baixo rendimento nas atividades diárias; modificações da rotina; falta de concentração; alterações de sono, transtornos psicológicos e medo.

Palavras-chave: COVID-19; Preditores estressantes; Problemas psicológicos.

\begin{abstract}
Considering the current global situation, marked by major crises in public health and, more recently, the pandemic caused by COVID-19, the present study aimed to analyze the biopsychosocial changes resulting from the social isolation caused by the Pandemic in dentistry students from University of Pernambuco. Regarding the methodology, it was a field survey of quantitative analysis, cross-sectional and descriptive. The sample consisted of a universe of 379 students, 106 of whom participated in the study. The collection was carried out over a period of 72 hours, in June 2021, where students answered an online questionnaire developed through the Google Forms platform containing closed and open questions about sociodemographic data, as well as aspects related to physical and psychosocial changes. The results suggest that in the sample studied, social isolation had repercussions on the daily routine, many reported problems with concentration, sleep disorders, feelings of fear, worry and sadness. It was concluded that the majority of respondents were female, the prevalent age group was 18 to 24 years old, they live with 2 or 4 more people in their homes, are single and reported having had financial losses with the pandemic. Among the main psychosocial issues, the following stood out: low performance in daily activities; routine modifications; lack of concentration; sleep disorders, psychological disorders and fear.
\end{abstract}

Keywords: COVID-19; Stressful predictors; Psychological problems.

\title{
Resumen
}

Considerando la situación global actual, marcada por grandes crisis de salud pública y, más recientemente, la pandemia provocada por COVID-19, el presente estudio tuvo como objetivo analizar los cambios biopsicosociales resultantes del aislamiento social provocado por la Pandemia en estudiantes de Odontología de la Universidad de Barcelona. Pernambuco. En cuanto a la metodología, se trató de una encuesta de campo de análisis cuantitativo, transversal y descriptivo. La muestra estuvo conformada por un universo de 379 estudiantes, de los cuales 106 participaron en el estudio. La recolección se llevó a cabo durante un período de 72 horas, en junio de 2021, donde los estudiantes respondieron un cuestionario en línea desarrollado a través de la plataforma Google Forms que contenía preguntas cerradas y abiertas sobre datos sociodemográficos, así como aspectos relacionados con cambios físicos y psicosociales. Los resultados sugieren que en la muestra estudiada, el aislamiento social repercutió en la rutina diaria, muchos reportaron problemas de concentración, trastornos del sueño, sentimientos de miedo, preocupación y tristeza. Se concluyó que la mayoría de los encuestados eran mujeres, el grupo de edad predominante era de 18 a 24 años, viven con 2 o 4 personas más en sus hogares, son solteros y reportaron haber tenido pérdidas económicas con la pandemia. Entre los principales problemas psicosociales se destacaron los siguientes: bajo desempeño en las actividades diarias; modificaciones de rutina; falta de concentración; trastornos del sueño, trastornos psicológicos y miedo.

Palabras clave: COVID-19; Predictores estressantes; Problemas psicológicos.

\section{Introdução}

As pandemias são conhecidas como epidemias que se dissipam por vários países, atingem uma grande quantidade de pessoas e geram consequências em todos os sistemas da sociedade. Trazem consigo novas regras e hábitos sociais e a necessidade de uma mobilização de naturezas diversas para conte-las. O surto da COVID-19 se espalhou por todos os continentes, causando perdas econômicas, diminuição da interação física e um sofrimento psicológico significativo (Duarte et al., 2020).

Desde 2019 a China e os países dos demais continentes estão lutando contra o SARS-Cov-2, que é um betacoronavírus onde sua transmissão é de humano para humano e observada por meio de gotículas respiratórias contaminadas em contato com a conjuntiva, mucosa nasal e bucal. A transmissibilidade, indicada pelo seu número reprodutivo foi estimada em 4,08 se tratando assim de uma patologia altamente contagiosa (Bezerra et al., 2020; Jakovljevic et al., 2020).

A pandemia teve como primeiro centro a cidade de Wuhan, China, no começo de dezembro de 2019. Em março do ano seguinte a Organização mundial de saúde (OMS) declarou a Europa como novo centro da pandemia. A COVID-19 foi anunciada pela OMS como emergência internacional da saúde pública em 30 de janeiro de 2020. Naquele momento, havia 7,7 mil casos confirmados e 170 óbitos na China, principal local de disseminação do vírus, e 98 casos em outros 18 países. No 
Brasil, nove casos estavam sendo investigados na época. O Ministério da Saúde recebeu a primeira notificação de um caso confirmado de Covid-19 no país em 26 de fevereiro de 2020. Com base nos dados diários informados pelas Secretarias Estaduais de Saúde ao Ministério da Saúde, de 26 de fevereiro de 2020 a 24 de julho de 2021, foram confirmados 19.670.534 casos e 549.448 óbitos por Covid-19 no Brasil. Em 05 de março de 2020 foi registrado os primeiros casos no estado de Pernambuco. (Brasil, 2021; Dubey et al., 2020; Jakovljevic et al., 2020; Pernambuco, 2020).

Pelo alto poder de contágio da COVID-19, os sistemas de saúde vêm enfrentando grandes desafios na tentativa de conter o número de infectados, identificar e administrar os casos e garantir estratégias eficazes para proteger a saúde pública. Esses desafios, embora principalmente surjam de uma doença infecciosa com implicações para a saúde física, têm atravessado todo o tecido social, não poupando praticamente nenhuma área da vida coletiva ou individual, tais desafios trouxeram repercussões profundas na esfera da saúde mental (Hossain et al., 2020; Lima, 2020).

Desde o surto, o isolamento social foi indicado pelos governos como uma das medidas de saúde pública para o controle da disseminação da COVID-19. Apesar de ser uma medida com eficácia, esse distanciamento influenciou direta ou indiretamente o bem estar psicológico da população. Segundo Lima (2020) em uma circunstância de epidemia, o número de pessoas psicologicamente afetadas tende a ser maior que o de pessoas acometidas pela infecção. Estudos recentes revelam que depressão, transtornos de ansiedade, transtornos de humor, sintomas de estresse pós-traumático, distúrbios do sono, pânico, baixa autoestima, falta de autocontrole são altamente prevalentes entre os indivíduos impactados pelo isolamento social. As mudanças no padrão de convivência vêm despertando sentimentos de solidão, medo, ansiedade generalizada, temor pela alta taxa de transmissão e até um estigmatização e discriminação de pessoas infectadas. Outra revisão sugeriu que fatores de estresse como quarentena prolongada, medo de infecção, frustração, tédio, suprimentos inadequados, informações inadequadas, perda financeira resultaram em sintomas de estresse pós-traumático de longa duração, confusão e raiva na população em massa (Bezerra et al., 2020; Hossain et al., 2020; Lima, 2020).

A vida pós pandemia não será igual. Assim, perguntas diversas são pertinentes em todos os aspectos que perpassam os seres humanos e a sociedade. Dentro desse contexto, essa pesquisa teve como objetivo verificar alterações biopsicossociais decorrentes do isolamento social no curso da pandemia em um grupo de alunos do curso de Odontologia da Universidade de Pernambuco e caracterizar a amostra pesquisada.

\section{Metodologia}

Tratou-se de um levantamento de campo (survey) de análise quantitativa, de corte transversal e descritivo. Segundo Gil (2008) "pesquisas deste tipo se caracterizam pela interrogação direta das pessoas cujo comportamento se deseja conhecer [...] procede-se à solicitação de informações a um grupo significativo de pessoas acerca do problema estudado para [...] obter as conclusões correspondentes dos dados coletados" (p. 55). Realizado com os alunos da Faculdade de Odontologia da Universidade de Pernambuco campus Camaragibe no período 7 a10 de junho de 2021. Foi constituída de um universo de 379 alunos regularmente matriculados no curso de Odontologia do $3^{\circ}$ ao $10^{\circ}$ período, com a participação de 106 alunos. Foram incluídos maiores de 18 anos que quiseram participar e tiveram esclarecimentos sobre a pesquisa e assinaram o Termo de Consentimento Livre e Esclarecido (TCLE) e excluído os demais alunos que não quiseram participar. Foi utilizado um questionário on-line desenvolvido por meio da plataforma Google Forms contendo perguntas fechadas e abertas sobre dados sociodemográficos para caracterização da amostra, bem como aspectos relacionados a alterações físicas e psicossociais. O questionário já foi validado por Bezerra et al. (2020) e Duarte et al. (2020) e aplicado para esse estudo. A coleta de dados ocorreu publicamente pelas redes sociais da pesquisadora disponibilizada por meio das plataformas Whats App e Instagram. Para isto houve uma divulgação prévia nos grupos do Whats App das turmas e pelo Instagram foi publicada uma caixa de perguntas e pelas respostas era enviado o link do formulário. A coleta foi realizada em um período de 72 horas, nos dias 7 a10 
de junho de 2021. Por se tratar de uma pesquisa pública os princípios éticos seguiram as Normas da Resolução CNS/MS, $\mathrm{n}^{\circ}$ 466/2012 e 510/2016, não sendo necessário, portanto, a submissão ao Comitê de Ética e Pesquisa (CEP/CONEP). Contudo, seguiram-se os cuidados e preceitos éticos que norteiam as pesquisas científicas do Conselho Nacional de Saúde (Brasil, 2016). Os dados foram analisados por meio do Microsoft Excel que possibilitou a utilização de Estatística descritiva, apresentando os resultados em forma de gráficos e tabelas das variáveis estudadas nesse estudo.

\section{Resultados}

A amostra foi constituída por um total de 106 alunos, sendo predominantemente do sexo feminino (92; 86,8\%), na faixa etária de 18 a 24 anos $(84 ; 79,2 \%)$ e estado civil solteiro (99; 93,4\%). Sobre a renda dos participantes quando questionados sobre o impacto econômico gerado pela pandemia, se a renda familiar havia sido reduzida 52,8\% (56) referiu ter tido perdas econômicas nesse período. 90,6\% (96) responderam que moram com 2 a 4 pessoas em seu domicílio (Tabela 1).

Tabela 1 - Características sociodemográficas.

\begin{tabular}{|c|c|c|}
\hline Variáveis & $\mathbf{N}$ & $\%$ \\
\hline \multicolumn{3}{|l|}{ Sexo } \\
\hline Masculino & 14 & 13,2 \\
\hline Feminino & 92 & 86,8 \\
\hline \multicolumn{3}{|l|}{ Faixa etária } \\
\hline 18 a 24 anos & 84 & 79,2 \\
\hline 25 a 31 anos & 22 & 20,8 \\
\hline \multicolumn{3}{|l|}{ Estado civil } \\
\hline Solteiro(a) & 99 & 93,4 \\
\hline Casado(a) & 7 & 6,6 \\
\hline Divorciado(a) & 0 & 0 \\
\hline Viúvo(a) & 0 & 0 \\
\hline \multicolumn{3}{|c|}{$\begin{array}{l}\text { Você teve renda reduzida em decorrência da } \\
\text { pandemia da covid-19 }\end{array}$} \\
\hline Sim & 56 & 52,8 \\
\hline Não & 50 & 47,2 \\
\hline \multicolumn{3}{|c|}{ Quantas pessoas moram no seu domicílio } \\
\hline Moro sozinho(a) & 1 & 0,9 \\
\hline 2 a 4 pessoas & 96 & 90,6 \\
\hline 5 a 8 pessoas & 9 & 8,5 \\
\hline Mais de 9 pessoas & 0 & 0 \\
\hline
\end{tabular}

Fonte: Autores

No que concerne os fatores associados às alterações biopsicossociais durante a pandemia da COVID-19 90,6\% (96) dos participantes referiram medo de ser infectado pela COVID-19; 88,7\% (94) relataram preocupação ao sair de casa; 67\% (71) informaram que moram na mesma casa que pessoas do grupo de risco; 63,2\% (67) dos participantes informaram que estão praticando o distanciamento ou estão saindo apenas para o essencial. Os participantes puderam assinalar mais de uma opção sobre a procura a diferentes tipos de informações sobre a COVID-19, os indivíduos reportaram índices semelhantes no acesso à informação sobre número de infectados e mortes e sobre autocuidado e prevenção. 
Tabela 2 - Fatores associados as alterações biopsicossociais durante a pandemia da COVID-19.

\begin{tabular}{|c|c|c|}
\hline Variáveis & $\mathbf{N}$ & $\%$ \\
\hline \multicolumn{3}{|c|}{ Você tem medo de ser infectado pela covid-19? } \\
\hline $\operatorname{Sim}$ & 96 & 90,6 \\
\hline Não & 10 & 9,4 \\
\hline \multicolumn{3}{|c|}{$\begin{array}{l}\text { Sente-se preocupado se você ou alguém do seu } \\
\text { domicílio precisa sair de casa? }\end{array}$} \\
\hline Sim & 94 & 88,7 \\
\hline Não & 12 & 11,3 \\
\hline \multicolumn{3}{|c|}{$\begin{array}{l}\text { Você mora na mesma casa que pessoas do } \\
\text { grupo de risco para o COVID-19? }\end{array}$} \\
\hline $\operatorname{Sim}$ & 71 & 67 \\
\hline Não & 35 & 33 \\
\hline \multicolumn{3}{|c|}{$\begin{array}{l}\text { Neste momento, você está em distanciamento } \\
\text { por indicação médica ou saindo de casa apenas } \\
\text { para o essencial? }\end{array}$} \\
\hline Sim & 67 & 63,2 \\
\hline Não & 39 & 36,8 \\
\hline \multicolumn{3}{|c|}{$\begin{array}{l}\text { Você procura informações em relação ao novo } \\
\text { coronavírus? } *\end{array}$} \\
\hline $\begin{array}{l}\text { Sobre número de infectados } \\
\text { e mortes }\end{array}$ & 64 & 60,4 \\
\hline Sobre prevenção e & 78 & 73,6 \\
\hline \multicolumn{3}{|l|}{ Autocuidado } \\
\hline Não procuro & 14 & 13,2 \\
\hline
\end{tabular}

*Pergunta com possibilidade de múltipla escolha. Fonte: Autores.

Ainda sobre os fatores associados as alterações biopsicossociais 71,7\% (76) dos respondentes declararam que o período de isolamento social modificou sua rotina mas conseguiram se adaptar; 62,3\% (66) estão fazendo atividades para lidar coma situação - como atividade física, práticas religiosas, ações lúdicas e 34,8\% (37) procuraram ajuda profissional; 60,4\% (64) já receberam diagnóstico de transtorno mental; 56,6\% (60) perceberam que o padrão do sono modificou e 67,5\% (56) deles informaram que isso tem dificultado o desempenho nas atividades diárias; a grande maioria dos participantes (94; 88,7\%) referiram uma dificuldade de se concentrar nas atividades do dia a dia ou com a sensação de "branco na mente". Observa-se que em relação ao medo de ser contaminado pela COVID-19 90\% dos respondentes relatou sentir medo. Também foi relatado que $88,7 \%$ têm medo em relação aos que moram em seu domicílio de ser contaminado. 67,5\% responderam que seu desempenho diário foi alterado, bem como houve modificação na sua rotina $71,7 \%$. Em relação ao padrão de sono, $56,6 \%$ informaram que tiveram alterações. Foi respondido ainda, que 34,9\% quando sentiram tristeza ou desconforto emocional procurou ajuda médica (Tabela 3). 
Tabela 3 - Fatores associados as alterações biopsicossociais durante a pandemia da COVID-19.

\begin{tabular}{|c|c|c|}
\hline Variáveis & $\mathbf{N}$ & $\%$ \\
\hline \multicolumn{3}{|l|}{ O período de isolamento social interferiu na sua rotina? } \\
\hline Não interferiu & 0 & 0 \\
\hline $\begin{array}{l}\text { Modificou minha rotina, mas } \\
\text { consegui me adaptar }\end{array}$ & 76 & 71,7 \\
\hline $\begin{array}{l}\text { Modificou minha rotina e não } \\
\text { consegui me adaptar }\end{array}$ & 30 & 28,3 \\
\hline \multicolumn{3}{|c|}{ Caso se sinta preocupado ou triste, como está fazendo para } \\
\hline $\begin{array}{l}\text { Procurei ajuda profissional/ } \\
\text { Psicóloga }\end{array}$ & 37 & 34,9 \\
\hline Tenho utilizado medicamentos & 25 & 23,6 \\
\hline Tenho feito outras atividades & 66 & 62,3 \\
\hline Ainda não pensei numa resolução para esta questão & 25 & 23,6 \\
\hline \multicolumn{3}{|c|}{$\begin{array}{l}\text { Alguma vez já deram a você algum diagnóstico de transtorno } \\
\text { mental? }\end{array}$} \\
\hline $\operatorname{Sim}$ & 64 & 60,4 \\
\hline Não & 42 & 39,6 \\
\hline \multicolumn{3}{|c|}{$\begin{array}{l}\text { Após o início do isolamento social, o seu padrão de sono } \\
\text { modificou? }\end{array}$} \\
\hline Sim & 60 & 56,6 \\
\hline Não & 46 & 43,4 \\
\hline \multicolumn{3}{|c|}{$\begin{array}{l}\text { Se sim, estes sintomas têm dificultado o seu desempenho nas } \\
\text { atividades diárias? }\end{array}$} \\
\hline Sim & 56 & 67,5 \\
\hline Não & 16 & 19,3 \\
\hline Não informado & 11 & 13,3 \\
\hline \multicolumn{3}{|c|}{$\begin{array}{l}\text { Durante o isolamento social, tem sentido dificuldade de se } \\
\text { concentrar nas atividades do dia a dia ou com a sensação de } \\
\text { "branco na mente"? }\end{array}$} \\
\hline Sim & 94 & 88,7 \\
\hline Não & 12 & 11,3 \\
\hline
\end{tabular}

*Pergunta com possibilidade de múltipla escolha. Fonte: Autores.

A Tabela 4 exprime os resultados da análise bivariada referente ao fato de o participante estar ou não em isolamento social. Observam-se diferenças significantes entre o fato de o respondente estar ou não em isolamento social nas seguintes variáveis: as pessoas que estão em isolamento social sentiam mais medo de serem infectadas pelo novo coronavírus, exprimiram maior preocupação se alguém precisa sair de casa e também relataram mudança no padrão de sono. Os participantes que estão em distanciamento relataram sentir dificuldade de se concentrar nas atividades do dia a dia ou com a sensação de "branco na mente" (Tabela 4). 
Tabela 4 - Comparativo entre pessoas que estão ou não em isolamento social durante a pandemia da covid-19.

\section{Variáveis}

Você tem medo de ser infectado pela covid-

19 ?

Sim

Não

Sente-se preocupado se você ou alguém do seu domicílio precisa sair de casa?

Sim

Não

Você mora na mesma casa que pessoas do grupo de risco para o COVID-19?

Sim

Não

Alguma vez já deram a você algum diagnóstico de transtorno mental?

Não

Após o início do isolamento social, o seu padrão de sono modificou?

Sim

Não

Tem sentido algum sintoma físico sem motivo aparente durante $o$ isolamento social?

Sim

Não

Durante o isolamento social, tem sentido

dificuldade de se concentrar nas atividades

do dia a dia ou com a sensação de "branco na mente"?

Sim

Não

O período de isolamento social interferiu na sua rotina?

Modificou minha rotina, mas consegui me adaptar

Modificou minha rotina e não consegui me adaptar

Você procura informações em relação ao

novo coronavírus? *

Sobre número de infectados

e mortes

Sobre prevenção e

Autocuidado

Sobre número de infectados e mortes e sobre prevenção e autocuidado

Não procuro

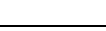

\section{Está em Isolamento Não está em Isolamento}

\begin{tabular}{|c|c|c|c|}
\hline \multicolumn{2}{|c|}{ Social } & \multicolumn{2}{|c|}{ Social } \\
\hline $\mathbf{N}$ & $\%$ & $\mathbf{N}$ & $\%$ \\
\hline 62 & 93 & 34 & 87 \\
\hline 5 & 7 & 5 & 13 \\
\hline
\end{tabular}

$62 \quad 93$

$\begin{array}{ll}32 & 82 \\ -7 & \end{array}$

$\begin{array}{llll}5 & 7 & 7 & 18\end{array}$

$\begin{array}{llll}48 & 72 & 23 & 59 \\ 19 & 28 & 16 & 41\end{array}$

41

18


86,6\% em relação ao masculino com 13,2\% e a faixa etária predominante na amostra foi de 18 a 24 anos com percentual de 79,2\%. Esses resultados são respaldados nos trabalhos de Duarte et al. (2020), Kuehner (2017) e Venkatesh e Edirappuli (2020), onde os autores relataram em suas pesquisas, que a maior prevalência de alterações psicossociais encontradas é em mulheres e na faixa etária mais nova, e esta faixa etária é extremamente vulnerável aos preditores de estresse.

Os resultados desse estudo apontaram que $52,8 \%$ dos pesquisados disseram que tiveram perdas financeiras advindas com a pandemia. A literatura mundial afirma que estas perdas, interferem emocionalmente gerando estresse, ansiedade, medo em relação ao futuro e podem provocar sofrimento psíquico levando a uma série de desestruturações emocionais. Corroboram com esses resultados os trabalhos de Brooks et al. (2020), Schmidt et al. (2020) e Tassara et al. (2020). Considerando que a saúde física e mental de uma população recebe influência do contexto socioeconômico, mercado de trabalho, assistência social e políticas públicas, é possível considerar que mudanças nessas dimensões reflitam nos indicadores de bem-estar da mesma, especialmente quando já há uma prevalência anterior de transtornos mentais (Schmidt et al., 2020).

Podem ser observados na Tabela 2, alguns fatores associados às alterações biopsicossociais que têm ocorrido durante a pandemia da Covid-19. O sentimento de medo de ser infectado pela Covid teve um percentual de 90,6\%. Esse resultado é preocupante porque o indivíduo em uma situação de estresse permanente, facilmente entrará em sofrimento psíquico e as alterações psíquicas surgirão e terão efeito severo na sua qualidade de vida (Boshra et al., 2020; Hossain et al., 2020; Lima, 2020; Wang et al., 2020). Segundo Enumo et al.(2020) os indicadores mais comuns de estresse e ansiedade durante períodos de isolamento social demorado são de ordem: física, como aumento da frequência cardíaca, alteração no padrão de sono e alimentação, exaustão, dor de cabeça, úlceras; emocionais, como nervosismo, aborrecimento, tristeza, culpa, humor deprimido, preocupação excessiva; comportamentais, como irritabilidade, abuso de substâncias, violência; cognitivos, como perda de memória, concentração debilitada e indecisão.

Entre os pesquisados desse estudo, o sentimento de preocupação em sair de casa apresentou um percentual de 88,7\% essa percepção elevada de medo de se contaminar pela COVID-19 advêm de vários fatores e diversas razões podem ter contribuído para isso, entre elas as informações divulgadas pela mídia e dramaticamente propagadas pelas redes sociais, desde os primeiros casos no país, sobre a iminente ameaça de propagação do vírus, o elevado número de casos e de mortes em outros países e o fato de não existir vacina ou opções comprovadas de tratamento antiviral específico. Esses resultados são enfatizados nos estudos de Bichara (2020) e Kalil (2020). Convém ressaltar um dado relevante encontrado nesse estudo, onde a amostra pesquisada teve um percentual $63,2 \%$ de respostas, que diziam que se mantinham em isolamento social por indicação médica. Esse resultado sugere que: ou os pesquisados têm alguma comorbidade ou desenvolveram alterações psicossociais. Estudos têm demonstrado que o medo de ser infectado por um patógeno potencialmente fatal e cujas origens e curso são desconhecidos afeta a saúde mental de muitos indivíduos podendo levar a um aumento de quadros de depressão, ansiedade e estresse em momentos de pandemia. Esse resultado está de acordo com a pesquisa de Bichara, Kalil e Schmidt et al. (2020). Estudos diversos realizados com a população chinesa durante a atual pandemia encontrou que a exposição frequente a mídias sociais e notícias sobre a COVID-19 estaria associada a mais chances de risco para sintomas de ansiedade e de depressão, o que se manteve mesmo quando controlados outros fatores. Sobre esse tópico, o diretor-geral da OMS afirmou que o mundo está enfrentando não apenas uma pandemia, mas também uma "infodemia", indicando a exposição prolongada e excessiva a informações sobre um problema, o que torna difícil o vislumbre de uma solução. Com isso, também muita desinformação e rumores podem ser espalhados durante uma emergência de saúde, o que pode dificultar uma resposta eficaz à saúde pública e criar confusão e desconfiança entre as pessoas (Duarte et al., 2020).

É importante relatar nesse estudo, que entre os pesquisados, o percentual de diagnóstico sobre problemas emocionais antes da pandemia foi de 60,4\%. Esse dado é preocupante. Diante de uma pandemia como essa que o mundo enfrenta já ter alguma alteração psíquica, requer cuidados especializados para se evitar o estresse pós traumático advindo após a pandemia, 
pois, já existe uma fragilidade emocional que poderá ser agravada com os novos preditores de estresse advindos com a crise epidemiológica da Covid 19. De acordo com Usher et al. (2020) pessoas com algum transtorno mental grave pode experimentar uma exacerbação de sintomas pré-existentes como resultante de um desastre (Duarte et al., 2020; Kavoor, 2020; Kuehner, 2017).

O conceito de saúde mental é complexo, não se trata somente da ausência de perturbação ou problema ou na manifestação de sofrimento mental, mas ele também é entendido como o produto de diversas interações sociais, biológicas, econômicas, culturais, psicológicas. Todo ser humano pode possuir algum incômodo que tenha impactado na sua saúde mental e para se caracterizar como de fato um problema de saúde mental deve-se observar se a intensidade e frequência ameaçam o bem-estar de quem o experiencia. $\mathrm{O}$ contexto pandêmico e suas respectivas medidas de controle vêm afetando a população em muitas dimensões, entre elas, de forma bastante significativa, a área de saúde mental. Algumas características tais como a presença de transtornos mentais, o sofrimento psíquico contínuo e a alteração do sono vêm exercendo efeitos negativos na qualidade da saúde e da vida das pessoas. Ao longo do desenvolvimento humano a psicologia reuniu um conjunto de conhecimentos sobre o controle das emoções e o enfrentamento adaptativo do estresse. A literatura define como situações estressoras aquelas às quais o indivíduo tem dificuldade para lidar, pois estão além da capacidade de enfrentamento, ou aquela na qual ele se avalia como incapaz de lidar com os conflitos internos gerados pelo evento em si. (Pavani et al., 2021; Barros et al., 2020; Enumo et al.,2020).

Ficou evidenciado na tabela 3, o comparativo de respostas dos pesquisados que estavam em isolamento com os que não aderiram ao isolamento. Ressalta-se que os dados obtidos merecem uma análise criteriosa: Por que os que estavam em isolamento e os que não estavam à diferença das respostas não foram significativas? Os estudos desde 2020 falam do caráter estressor do isolamento e alterações biopsicossociais (Brooks et al., 2020; Lima et al., 2020; Ozili e Arun, 2020; Schmidt et al., 2020; Tassara et al., 2021; Wang et al., 2020).

Um dado obtido na tabela 3 sobre a interferência do isolamento na rotina diária apresentou nos pesquisados que estava em isolamento um percentual de 70\%, mas, os que não estavam em isolamento foi obtido um percentual de $74 \%$. Esse resultado não encontra respaldo na literatura consultada no presente estudo. Também em relação a capacidade de concentração nas atividades diárias dos que estavam em isolamento foi de $90 \%$, mas, os que não estavam em isolamento tiveram também um percentual elevado que foi de $87 \%$. Esses dados sugerem que a questão das alterações psicossociais advindas da pandemia não tem ainda estudos comparativos em relação a isolamento social, pois, diante da gravidade da pandemia, as pesquisas sobre o patógeno, questões sobre vacinação e seus protocolos de tratamento são mais estudados no momento.

\section{Considerações Finais}

Conclui-se que entre as principais questões psicossociais do presente estudo destacaram-se: alterações físicas, baixo rendimento nas atividades diárias; modificações da rotina cotidiana; falta de concentração; alterações de sono, transtornos psicológicos, tristeza e medo. A maioria dos pesquisados foi do gênero feminino, a faixa etária prevalente foi de 18 a 24 anos; moram com mais 2 ou 4 pessoas em seus domicílios; são solteiros e relataram terem tido perdas financeiras com a pandemia. Ressalta-se como ponto relevante desse estudo, a importância de trazer à tona que a pandemia é um preditor de alterações psicossociais. E toda informação a esse respeito ajudará políticas preventivas de saúde mental diante e pós pandemia. Também cabe mencionar como limitação desse estudo, a utilização de uma amostra de conveniência, predominantemente feminina e com pesquisados de maior escolaridade. Assim, mais pesquisas precisam ser desenvolvidas sobre o tema. Uma pandemia é uma catástrofe mundial que deverá ser estudada em todos os seus aspectos para trazer respostas no sentido de atenuar os danos causados. 


\section{Referências}

Barros, M. B. D. A., Lima, M. G., Malta, D. C., Szwarcwald, C. L., Azevedo, R. C. S. D., Romero, D., \& Gracie, R. (2020). Relato de tristeza/depressão, nervosismo/ansiedade e problemas de sono na população adulta brasileira durante a pandemia de COVID-19. Epidemiologia e Serviços de Saúde, 29, e2020427.

Bezerra, C. B., Saintrain, M. V. D. L., Braga, D. R. A., Santos, F. D. S., Lima, A. O. P., Brito, E. H. S. D., \& Pontes, C. D. B. (2020). Impacto psicossocial do isolamento durante pandemia de covid-19 na população brasileira: análise transversal preliminar. Saúde e Sociedade, 29 , e200412.

Bichara, M. (2020). SARS-COV-2 infodemia, pós-verdade e guerra híbrida. Revista Estudos Libertários, 2(3), 90-101.

Boshra, A. A., Al-Dabbagh, Z. S., Al Eid, N. A., Al Eid, M. A., Al-Musaibeh, S. S., Al-Miqtiq, M. N., \& Al-Zeyad, G. M. (2020). The effects of corona virus (COVID-19) outbreak on the individuals' mental health and on the decision makers: A comparative epidemiological study. International Journal of Medical Research \& Health Sciences, 9(3), 26-47.

Brasil. Ministério da Saúde. Secretaria de Vigilância em Saúde. Boletim Epidemiológico Especial Doença pelo Coronavírus COVID-19 N73. (2021)

Brooks, S. K., Webster, R. K., Smith, L. E., Woodland, L., Wessely, S., Greenberg, N., \& Rubin, G. J. (2020). The psychological impact of quarantine and how to reduce it: rapid review of the evidence. The lancet, 395(10227), 912-920.

Duarte, M. D. Q., Santo, M. A. D. S., Lima, C. P., Giordani, J. P., \& Trentini, C. M. (2020). COVID-19 e os impactos na saúde mental: uma amostra do Rio Grande do Sul, Brasil. Ciência \& Saúde Coletiva, 25, 3401-3411.

Dubey, S., Biswas, P., Ghosh, R., Chatterjee, S., Dubey, M. J., Chatterjee, S., \& Lavie, C. J. (2020). Psychosocial impact of COVID-19. Diabetes \& Metabolic Syndrome: Clinical Research \& Reviews, 14(5), 779-788.

Enumo, S. R. F., Weide, J. N., Vicentini, E. C. C., Araujo, M. F. D., \& Machado, W. D. L. (2020). Enfrentando o estresse em tempos de pandemia: proposição de uma cartilha. Estudos de Psicologia (Campinas), 37.

Gil, A. C. (2008). Métodos e técnicas de pesquisa social. (6a ed.), Ediitora Atlas SA.

Hossain, M. M., Tasnim, S., Sultana, A., Faizah, F., Mazumder, H., Zou, L., \& Ma, P. (2020). Epidemiology of mental health problems in COVID-19: a review. F1000Research, 9.

Jakovljevic, M., Bjedov, S., Jaksic, N., \& Jakovljevic, I. (2020). COVID-19 pandemia and public and global mental health from the perspective of global health security. Psychiatria Danubina, 32(1), 6-14.

Kalil, I., \& Santini, R. M. (2020). Coronavírus, pandemia, infodemia e política. Relatório de pesquisa. Divulgado em, 1.

Kavoor, A. R. (2020). COVID-19 in people with mental illness: Challenges and vulnerabilities. Asian Journal of Psychiatry, 51 , 102051.

Kim, U.; \& Bhullar, N. Life in the pandemic: Social isolation and mental health. https://onlinelibrary. wiley. com/doi/pdfdirect/10.1111/jocn. 15290, 2020.

Kuehner, C. (2017). Why is depression more common among women than among men? The Lancet Psychiatry, 4(2), $146-158$.

Lima, C. K. T., de Medeiros Carvalho, P. M., Lima, I. D. A. A. S., de Oliveira Nunes, J. V. A., Saraiva, J. S., de Souza, R. I., \& Neto, M. L. R. (2020). The emotional impact of Coronavirus 2019-nCoV (new Coronavirus disease). Psychiatry research, 287, 112915.

Lima, R. C. (2020). Distanciamento e isolamento sociais pela Covid-19 no Brasil: impactos na saúde mental. Physis: Revista de Saúde Coletiva, 30.

Ozili, P. K., \& Arun, T. (2020). Spillover of COVID-19: impact on the Global Economy.

Pavani, F. M., Silva, A. B. D., Olschowsky, A., Wetzel, C., Nunes, C. K., \& Souza, L. B. (2021). Covid-19 and repercussions in mental health: a narrative review of literature. Revista gaúcha de enfermagem, 42.

Schmidt, B., Crepaldi, M. A., Bolze, S. D. A., Neiva-Silva, L., \& Demenech, L. M. (2020). Saúde mental e intervenções psicológicas diante da pandemia do novo coronavírus (COVID-19). Estudos de Psicologia (Campinas), 37.

Tassara, I. G., Okabayashi, N. Y. T., Casaca, M. C. G., \& de Souza Veronez, F. (2021). Prevalência de sintomas psicológicos em tempos de isolamento social. Brazilian Journal of Health Review, 4(1), 1295-1309.

Wang, C., Pan, R., Wan, X., Tan, Y., Xu, L., Ho, C. S., \& Ho, R. C. (2020). Immediate psychological responses and associated factors during the initial stage of the 2019 coronavirus disease (COVID-19) epidemic among the general population in China. International journal of environmental research and public health, 17(5), 1729. 